\title{
Successful Coronary Artery Bypass Operation in a SARS-COV-2 Infected Patient with Acute Coronary Syndrome
}

\author{
Srikanth Yandrapalli ${ }^{1}$, Howard Cooper ${ }^{1}$, and Ramin Malekan ${ }^{1}$ \\ ${ }^{1}$ Westchester Medical Center
}

June 1, 2020

\begin{abstract}
The Coronavirus disease 2019 (COVID-19) pandemic caused by the severe acute respiratory syndrome coronavirus 2 (SARSCoV-2) is overwhelming healthcare resources and infrastructure worldwide. Cardiac surgical operating capacity during the COVID-19 pandemic is dramatically lower due to postponement or cancellation of elective or semi-urgent procedures. Earlier reports have demonstrated complicated post-operative courses and high fatality rates in patients undergoing emergent cardiothoracic surgery who were diagnosed post-operatively with COVID-19. These reports raise the possibility that active COVID-19 might precipitate a catastrophic pathophysiogical response to infection in the post-operative period and lead to unfavorable surgical outcomes. Hence, it is imperative to screen patients with SARS-CoV-2 infection prior to surgery and to carefully monitor them in the post-operative period to identify any early signs of active COVID-19. In this report, we present the successful outcome of coronary artery bypass grafting (CABG) operation in a patient with asymptomatic SARS-CoV-2 infection presenting with an acute coronary syndrome and requiring urgent surgical intervention. We employed a meticulous strategy to identify subclinical COVID-19 disease, and after confirming the absence of active disease, proceeded with the CABG operation. The patient outcome was successful with the absence of any overt COVID-19 manifestations in the post-operative period.
\end{abstract}

\section{Introduction}

Coronavirus disease 2019 (COVID-19) caused by the severe acute respiratory syndrome coronavirus 2 (SARS$\mathrm{CoV}-2$ ) is an ongoing pandemic. Earlier reports have demonstrated complicated post-operative courses and high fatality rates in patients undergoing emergent cardiothoracic surgery and diagnosed in the post-operative with COVID-19. ${ }^{1-4}$ We present the first report of a coronary artery bypass grafting operation (CABG) with a successful outcome in a patient with SARS-CoV-2 infection.

\section{Case presentation}

A 67-year-old female with a history of obesity, gastric acid reflux disease, and hiatal hernia repair presented with persistent chest pain of 1-day duration. Her husband had weakness and fatigue of 1-week duration. Laboratory testing is presented in Figure. Given exposure to her husband who had non-specific symptoms, nasal swab testing for the SARS-CoV-2 was done which returned positive. She did not have respiratory symptoms, hypoxia, or fevers suggestive of COVID-19 pneumonia. Chest radiography showed clear lung fields (Figure Panel A) . She was admitted for a non-ST elevation myocardial infarction. Cardiac catheterization revealed severe three-vessel coronary artery disease (CAD), a culprit lesion in a large branching first diagonal with complete occlusion and grade 2 left to left collaterals; diffuse, severe, proximal and mid-left anterior descending artery CAD, and severe proximal right coronary artery CAD. There was severe distal left circumflex and left posterior descending artery CAD. Echocardiography revealed normal ventricular function and wall motion. 
In the setting of continued symptoms from the acute coronary syndrome and multivessel CAD, cardiac surgical consultation was requested. Laboratory testing to identify inflammatory markers elevations associated with COVID-19 were normal; Figure. Computed tomographic (CT) chest imaging confirmed clear lung fields without pulmonary parenchymal pathology (Figure Panel B) . After ruling out active COVID-19 infection, we decided to proceed with CABG surgery.

Following uneventful induction of general anesthesia, aorta-bicaval cannulation was utilized for cardiopulmonary bypass. Myocardium was protected with cold blood cardioplegia. Coronary revascularization was performed with an in situ left internal mammary arterial graft to the left anterior descending artery, saphenous venous graft to the first diagonal, and saphenous venous graft to the left posterior descending artery. The patient was weaned from cardiopulmonary bypass without difficulty and transferred to the ICU. She was extubated within 4 hours of ICU admission and transitioned to nasal cannula oxygen at $2 \mathrm{~L} / \mathrm{min}$. Acute blood loss anemia was managed with transfusion\souts. Post-operative day 1 chest radiograph showed atelectasis which was managed with incentive spirometry and pain control. Subsequent radiographs showed the development of bilateral small pleural effusions (Figure Panel C), accompanied by the requirement for increase in nasal oxygen support to $5 \mathrm{~L} / \mathrm{min}$. The effusions were successfully treated with furosemide. The patient was then transferred to the step-down unit on post-operative day 4 . She was afebrile during this period with no significant laboratory abnormalities. Respiratory status continued to improve, with improvement in chest radiography findings and nasal oxygen support was gradually weaned off (Figure Panel D ). The patient was discharged home on post-operative day 6 .

\section{Discussion}

We present a case of a successful CABG surgery in a patient with asymptomatic SARS-CoV-2 infection presenting with acute coronary syndrome and with multi-vessel severe CAD requiring urgent surgery. We thoroughly evaluated the patient to identify active COVID-19 infection by assessing for common inflammatory markers and CT findings associated with COVID-19. After confirming the absence of subclinical COVID-19, we proceeded with the CABG operation with a favorable outcome and without post-operative COVID-19 manifestations.

To the best of our knowledge, there are no published reports of CABG operation in patients with preoperatively confirmed SARS-CoV-2 infection. In a report of post-operatively diagnosed COVID-19 after CABG for an acute coronary syndrome, the patient succumbed to severe pneumonia. ${ }^{1}$ In accordance with the United Kingdom National Health Service recommendations, the patient was not initially tested for the SARS-CoV-2 at presentation (15 days prior to surgery) as he did not have COVID-19 symptoms and had normal inflammatory markers. ${ }^{1}$ A chest CT scan at presentation showed mild chronic obstructive pulmonary disease. The patient developed pneumonia on postoperative day 1 with severe hypoxia and fever. Chest radiography showed bilateral consolidation and bronchial-alveolar lavage was positive for SARS-CoV-2. The authors recognized that undiagnosed or potentially concomitant COVID-19 infection might precipitate a catastrophic pathologic response refractory to standard treatments after cardiac surgery which might pose a challenge to clinicians. ${ }^{1}$ In a case series if eleven patients with COVID-19 infection diagnosed after lung cancer surgery, three patients expired in the immediate post-operative period. ${ }^{3}$ In two other reports of emergency thoracic surgery (embolectomy for pulmonary embolism and acute type A aortic dissection repair) and postoperatively diagnosed COVID-19, the patients had a complicated post-operative course and eventually died from multiorgan dysfunction. ${ }^{2,4}$ A review of a pre-operative CT chest in one of these patients showed signs consistent with COVID-19 lung lesions. ${ }^{2}$

The key takeaway points from our case presentation are: 1) Routine testing for the SARS-CoV-2 in all patients who require cardiac surgery to identify asymptomatic/subclinical infection. 2) In asymptomatic SARS-CoV-2 infection, assessing for subclinical active infection (with inflammatory markers and CT chest) helps to identify patients at higher risk of developing post-operative complications. This is especially important for patients requiring cardiopulmonary bypass as subclinical pneumonia can result in severe hypoxia and complicate weaning from cardiopulmonary bypass. 3) Careful monitoring of all SARS-COV-2 positive patients postoperatively for development of COVID-19. Nasal swab testing with reverse transcriptase- 
polymerase chain reaction to detect the SARS-CoV-2 RNA has only $70 \%$ sensitivity. Any clinical change to suggest active COVID-19 in the pre- or post-operative period should prompt repeat swab testing or chest CT imaging to improve risk stratification. Chest CT screening has a higher sensitivity (98\%) than nasal swab for SARS-CoV-2 infection as CT findings occur earlier. ${ }^{5}$ Post-intubation bronchoalveolar lavage can be considered.

As we move forward in this pandemic, we need to continue to provide appropriate medical care for patients with asymptomatic or subclinical SARS-COV-2 infection who require cardiac surgery. Careful screening strategies, standard guideline recommendations and hospital level protocols are required to identify higher risk patients who are at greater risk of post-surgical morbidity and mortality.

\section{References}

1. Rescigno G, Firstenberg M, Rudez I, Uddin M, Nagarajan K, Nikolaidis N. A Case of Postoperative Covid-19 Infection After Cardiac Surgery: Lessons Learned. Heart Surg Forum . 2020;23(2):E231-E233. doi:10.1532/hsf.3011

2. Fabre O, Rebet O, Carjaliu I, Radutoiu M, Gautier L, Hysi I. Severe Acute Proximal Pulmonary Embolism and COVID-19: A Word of Caution.Ann Thorac Surg . Published online April 17, 2020. doi:10.1016/j.athoracsur.2020.04.005

3. Peng S, Huang L, Zhao B, et al. Clinical course of coronavirus disease 2019 in 11 patients after thoracic surgery and challenges in diagnosis. J Thorac Cardiovasc Surg . Published online April 10, 2020. doi:10.1016/j.jtcvs.2020.04.005

4. Fukuhara S, Rosati CM, El-Dalati S. Acute Type A Aortic Dissection during COVID-19 Outbreak. Ann Thorac Surg . Published online April 22, 2020. doi:10.1016/j.athoracsur.2020.04.008

5. Fang Y, Zhang H, Xie J, et al. Sensitivity of Chest CT for COVID-19: Comparison to RT-PCR. Radiology . Published online February 19, 2020:200432. doi:10.1148/radiol.2020200432

\section{Figure legend}

Laboratory and Radiographic findings. Panel A : Chest radiograph on presentation showing clear lung fields. Panel B: A chest computed tomographic image in the axial plane demonstrates clear lung parenchyma. Panel C: Post-operative day 3 radiograph showing bibasilar atelectasis and bilateral pleural effusions. Panel D:Chest radiograph prior to discharge demonstrated improved atelectasis and pleural effusions.

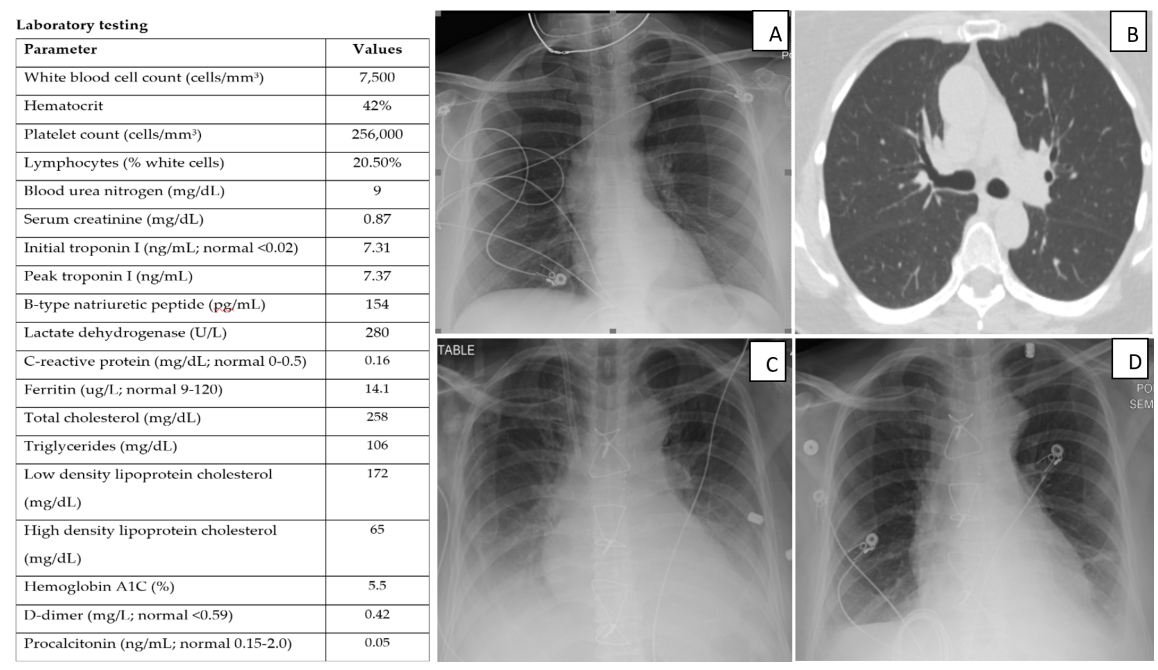

